\title{
Intraoperative radiotherapy for breast cancer: powerful evidence to change practice
}

\section{Jayant S. Vaidya $(D)$ Max Bulsara, Michael Baum, Michael Alvarado, Marcelle Bernstein, Samuele Massarut, Christobel Saunders, Elena Sperk, Frederik Wenz, Jeffrey S. Tobias and the TARGIT-A investigators}

We believe that the recent News and Views article (Sasieni, P. D. \& Sawyer, E. J. Intraoperative radiotherapy for early breast cancer insufficient evidence to change practice. Nat. Rev. Clin. Oncol. 17, 723-724 (2020)) $)^{1}$ about the TARGIT-A trial contains several factual and logical errors. This article overlooks both the long-term positive findings ${ }^{2}$ and the all-important patient perspective.

Risk-adapted single-dose targeted intraoperative radiotherapy during lumpectomy (TARGIT-IORT) is a method of partial breast irradiation (PBI) for early breast cancer. Most patients (80\%) receiving TARGITIORT $^{2}$ during their lumpectomy complete their local treatment entirely during this single session, under the same anaesthetic. Supplemental whole breast external beam radiotherapy (WBRT) is only recommended for a minority of patients (20\%) if unexpected prespecified tumour-related factors such as invasive lobular cancer and positive margins are found postoperatively. However, most patients with conventional 'high risk' features were treated without supplemental WBRT, including four-fifths of those with grade 3 or ER-negative disease, and twothirds of node-positive cases. By contrast, traditional WBRT or other PBI approaches require up to 30 additional hospital visits TARGIT-IORT involves far fewer clinic appointments ${ }^{3}$. Other benefits include fewer toxicities, less pain, better cosmetic results and better quality of life ${ }^{2}$.

The TARGIT-A randomized trial compared risk-adapted TARGIT-IORT with WBRT. The long-term results ${ }^{2}$ revealed no significant differences in local and distant control, breast preservation or breast cancer mortality. Local control was also comparable to that achieved with TARGIT-IORT alone $^{2}$. A significant reduction in non-breast cancer mortality (from cardiovascular causes and other cancers) was also observed with TARGIT-IORT, from $9.85 \%$ to $4.41 \%$ at 12 years $^{2}$. For patients, who sit on the more uncomfortable side of the consultation desk, these are most welcome results, particularly in the COVID-19 era.
The authors complain ${ }^{1}$ that TARGITIORT was not compared with 'no radiotherapy'; however, we emphasize that the TARGIT-A cohort had a much higher proportion of high-risk patients than trials investigating this approach (Supplementary information). In fact, more than three-quarters of patients $(1,737$ of 2,298$)$ in TARGIT-A ${ }^{2}$ would not have fulfilled the low-risk criteria for inclusion in a trial of 'no radiotherapy' such as PRIME-II (inclusion criteria: age $>65$ years, tumour diameter $\leq 3 \mathrm{~cm}$, grade 1 or 2, node-negative and ER positive). Despite this higher-risk cohort, local recurrence with TARGIT-IORT was 2-3 times lower than with 'no radiotherapy' in those trials (Supplementary information). Crucially, for a more inclusive population such as this, which is more representative of clinical practice, a 'no radiotherapy' arm would be unethical. We agree that "discriminating ... those who can safely avoid radiotherapy altogether remains a fundamental challenge" ${ }^{1}$, therefore, patients should not be recommended 'no radiotherapy necessary' without first discussing options such as TARGIT-IORT. We emphasize that with TARGIT-IORT completed during lumpectomy, $80 \%$ of patients do not need postoperative radiotherapy ${ }^{2}$.

The proportion of high-risk patients in the TARGIT-A cohort (PBI versus WBRT) is remarkably similar to that of the Fast-Forward cohort (shorter-course WBRT versus 3-week daily WBRT) (Supplementary information), which the authors recommend ${ }^{1}$. The 5-year local recurrence with 3-week WBRT in Fast-Forward and TARGIT-IORT was virtually identical at $2.1 \%$. If the authors ${ }^{1}$ seriously question whether TARGIT-IORT is better than 'no radiotherapy" ${ }^{1}$, should the same question not also apply to the Fast-Forward WBRT regimen? In any event, 'no radiotherapy' is not considered the standard of care for such patients and therefore is not the correct comparator.

The effectiveness of PBI approaches such as TARGIT-IORT has been repeatedly demonstrated (Supplementary information), yet the authors do not mention this important concept. Instead, they promote ${ }^{1}$ the intensive
'Fast-Forward' whole-breast-radiotherapy approach, which we argue represents overtreatment for the majority of patients and comes with well-known hazards: the most important adverse effects of an increased irradiated volume and the associated scattered irradiation are the substantially increased risks of cardiovascular ${ }^{4,5}$ and cancer-related mortality ${ }^{4,6}$, which are avoided by PBI techniques ${ }^{7}$ such as TARGIT-IORT ${ }^{2,8}$. Conversely, as expected with WBRT techniques, there is no mortality benefit with Fast-Forward. Fast-Forward also entails inevitable post-operative delay plus $7-15$ hospital visits (for consultation and planning followed by daily WBRT with or without boost).

The authors criticize the TARGIT-A noninferiority margin of $2.5 \%{ }^{2}$ and surprisingly claim ${ }^{1}$ that no radiotherapy (as used in PRIME-II, Supplemental information) is non-inferior to WBRT. We argue that the data disprove this claim - the actual difference in 5-year local recurrence in PRIME-II was $2.9 \%$, with an upper confidence interval of $4.8 \%$ - both well above the $2.5 \%$ margin $^{2}$. The $2.5 \%$ non-inferiority margin used in TARGIT- $\mathrm{A}^{2}$ is one of the most stringent (in both absolute and relative terms) among trials involving PBI (Supplementary information). Nonetheless, the actual difference in 5-year local recurrence between the two treatment arms of TARGIT-A was just $1.16 \%$.

The Kaplan-Meier model, which we used ${ }^{2}$ to analyse local control, includes all relevant events $^{9,10}$ in addition to time of occurrence and length of follow-up monitoring, for every patient. This is not the case for a chi-square test, which was employed by the authors ${ }^{1}$ to test for superiority, even though TARGIT-A was a non-inferiority trial - a very different concept: "Non-inferiority trials ... test new treatments that have obvious non-oncological advantages ... The non-inferiority statistical test ... is not meant to check for superiority, but to assess if the difference is within an acceptable margin and the experimental treatment is not meaningfully worse than the control" ${ }^{2}$. The protocol-specified non-inferior 5-year local recurrence associated with TARGIT-IORT was clearly confirmed in TARGIT-A.

Many countries across the world have enthusiastically embraced TARGIT-IORT, with $>45,000$ patients treated so far. TARGITIORT is now recommended in many international guidelines. Patient choice, informed by clearly presented evidence, is now recognized as being much more important than clinician preferences, a point powerfully underscored by the UK Supreme Court (Montgomery v Lanarkshire Health Board, 2015), the Royal College of Surgeons of England, and the UK General Medical Council. 
There is a reply to this letter by Sasieni, P. D. \& Sawyer, E. J. Nat. Rev. Clin. Oncol. https:// doi.org/10.1038/s41571-021-00472-6 (2020).

Jayant S. Vaidya (iD ${ }^{1 凶}$, Max Bulsara ${ }^{1,2}$, Michael Baum ${ }^{1}$, Michael Alvarado ${ }^{3}$ Marcelle Bernstein ${ }^{4}$, Samuele Massarut ${ }^{5}$ Christobel Saunders ${ }^{6}$, Elena Sperk ${ }^{7}$, Frederik Wenz ${ }^{8}$ Jeffrey S. Tobias ${ }^{9}$ and the TARGIT-A investigators *

'Division of Surgery and Interventional Science, University College London, London, UK. ${ }^{2}$ Department of Biostatistics, University of Notre Dame, Fremantle, WA, Australia.

${ }^{3}$ Department of Surgery, University of California San Francisco, CA, USA. ${ }^{4}$ London, UK.

${ }^{5}$ Department of Surgery, Centro di Riferimento Oncologico di Aviano (CRO) IRCCS, Aviano, Italy. ${ }^{6}$ School of Surgery, University of Western Australia, Perth, WA, Australia

${ }^{7}$ University Medical Center Mannheim, Medical Faculty Mannheim, Heidelberg University, Heidelberg, Germany.

${ }^{8}$ University Medical Center Freiburg, Freiburg, Germany. ${ }^{9}$ Department of Clinical Oncology, University College London Hospitals, London, UK *A list of authors and their affiliations appears at the end of the paper

凶e-mail: jayantvaidya@gmail.com https://doi.org/10.1038/s41571-021-00471-7

1. Sasieni, P. D. \& Sawyer, E. J. Intraoperative radiotherapy for early breast cancer - insufficient evidence to change practice. Nat. Rev. Clin. Oncol. 17, 723-724 (2020).

2. Vaidya, J. S. et al. Long term survival and local control outcomes from single dose targeted intraoperative radiotherapy during lumpectomy (TARGIT-IORT) for early breast cancer: TARGIT-A randomised clinical trial. BMJ 370, m2836 (2020).

3. Coombs, N. J. et al. Environmental and social benefits of the targeted intraoperative radiotherapy for breast cancer: data from UK TARGIT-A trial centres and two UK NHS hospitals offering TARGIT IORT BMJ Open 6 e010703 (2016)

4. Taylor, C. et al. Estimating the risks of breast cancer radiotherapy: evidence from modern radiation doses to the lungs and heart and from previous randomized trials. J. Clin. Oncol 35, 1641-1649 (2017).

5. Darby, S. C. et al. Risk of ischemic heart disease in women after radiotherapy for breast cancer. N. Engl. J. Med. 368, 987-998 (2013)

6. Grantzau, T. \& Overgaard, J. Risk of second nonbreast cancer after radiotherapy for breast cancer: a systematic review and meta-analysis of 762,468 patients. Radiother. Oncol. 114, 56-65 (2015).

7. Vaidya, J. S. et al. Reduced mortality with partial-breast irradiation for early breast cancer: a meta-analysis of randomized trials. Int. J. Radiat. Oncol. Biol. Phys. 96 259-265 (2016)

8. Aziz, M. H. et al. Can the risk of secondary cancer induction after breast conserving therapy be reduced using intraoperative radiotherapy (IORT) with low-energy x-rays? Radiat. Oncol. 6, 174 (2011).

9. Gourgou-Bourgade, S. et al. Guidelines for timeto-event end point definitions in breast cancer trials: results of the DATECAN initiative (Definition for the Assessment of Time-to-event Endpoints in CANcer trials). Ann. Oncol. 26, 873-879 (2015).

10. Hudis, C. A. et al. Proposal for standardized definitions for efficacy end points in adjuvant breast cancer trials: the STEEP system. J. Clin. Oncol. 25, 2127-2132 (2007).

\section{Acknowledgements}

Funding for the TARGIT-A trial was mainly provided by the National Institute for Health Research (NIHR) Health Technolog Assessment programme (HTA 07/60/49). J.S.V. and J.S.T received funding from the UK HTA, NIHR, and Department of Health for some activities related to the TARGIT trials.

\section{Competing interests}

J.S.V. has received research funding and honoraria from Photoelectron Corp and Carl Zeiss and has received travel support from Carl Zeiss. M. Bulsara has received travel support from Carl Zeiss. M. Baum has served on the scientific advisory board, has acted as a consultant of and received travel support from Carl Zeiss. M.A. has received travel

support from Carl Zeiss. M. Bernstein has received trave support from Carl Zeiss. S. M has received travel support from Carl Zeiss. C.S. has received travel support from Carl Zeiss. E.S. has received honoraria and travel support from Carl Zeiss. F.W. has received research funding and travel support from Carl Zeiss. J.S.T. has received travel support from Carl Zeiss.

\section{Supplementary information}

The online version contains supplementary material available at https://doi.org/10.1038/s41571-021-00471-7.

\section{The TARGIT-A investigators}

Jayant S. Vaidya ${ }^{1}$, Max Bulsara ${ }^{1,2}$, Michael Baum ${ }^{1}$, Michael Alvarado ${ }^{3}$, Marcelle Bernstein $^{4}$, Samuele Massarut ${ }^{5}$, Christobel Saunders ${ }^{6}$, Elena Sperk ${ }^{7}$, Frederik Wenz ${ }^{8}$ and Jeffrey S. Tobias ${ }^{9}$

A full list of consortium members and their affiliations appears in the supplementary information.

\section{Reply to 'Intraoperative radiotherapy for breast cancer: powerful evidence to change practice'}

\section{Peter D. Sasieni(1) and Elinor J. Sawyer (1)}

The TARGIT-A investigators claim in their Correspondence (Vaidya, J. S. et al. Intraoperative radiotherapy for breast cancer: powerful evidence to change practice. Nat. Rev. Clin. Oncol. https://doi.org/10.1038/ s41571-021-00471-7 (2020)) $)^{1}$ that our article (Sasieni, P. D. \& Sawyer, E. J. Intraoperative radiotherapy for early breast cancer - insufficient evidence to change practice. Nat. Rev. Clin. Oncol. 17, 723-724 (2020)) $)^{2}$ contains several factual and logical errors but we are unclear as to what these are. We stand by our assertion that the treatment that they recommend has not been shown to have any effect against local recurrence and suggest that, were radiotherapy held to the same regulatory standards as chemotherapy, this particular usage would not be licensed.

It seems that we failed to convey our central argument in that Vaidya et al. ${ }^{1}$ complain that we overlooked the long-term positive findings and the patient perspective. We accept that targeted intraoperative radiotherapy during lumpectomy (TARGIT-IORT) is more convenient than conventional whole-breast external beam radiotherapy (WBRT). However, a new treatment needs to have been shown (at least indirectly) to be superior to no treatment $^{3}$, and TARGIT-IORT fails in this regard. We know of no convincing argument that TARGIT-IORT might reduce non-breast cancer mortality relative to no radiotherapy, therefore, the question as to whether TARGIT-IORT results in fewer non-breast cancer deaths than WBRT is only relevant if TARGIT-IORT is shown to reduce breast cancer recurrence.

In their analysis of indirect evidence of superiority to no radiotherapy, the authors make an error - they focus on the wrong end of the confidence interval (CI). In Prime-II ${ }^{4}$ the $95 \% \mathrm{CI}$ for the additional risk of ipsilateral recurrence at 5 years comparing no radiotherapy with WBRT was $1.1-4.8 \%$. TARGIT-A has not shown the difference in local recurrence at 5 -years comparing TARGIT-IORT to WBRT to be $<1.1 \%$ (and may therefore be no better than no radiotherapy). Vaidya et al. make the point that patients enrolled in PRIME-II had a generally lower risk of recurrence, but 5-year local recurrence in women assigned WBRT was in fact higher in PRIME-II (1.3\%) than in TARGIT-A (1.0\%).

Rather than using the 95\% CI from a single trial, we would suggest using meta-analysis ${ }^{5}$ : we calculate the $95 \%$ CI for the risk ratio as 0.26-0.38 (Supplementary information). Thus, to show that TARGIT-IORT is superior to no radiotherapy, one would have to set a non-inferiority margin on the relative risk of $1 / 0.38=2.6$, and require that the upper limit of the 95\% CI comparing TARGIT-IORT with WBRT is at most 2.6. Based on the number of local recurrences by 5 years in the per-protocol analysis, the $95 \%$ CI for the risk ratio in TARGIT-A is 1.14-4.99 (REF.') (Supplementary information). By contrast, in FAST-Forward', the upper limit of the $95 \%$ CI for the hazard ratio comparing $26 \mathrm{~Gy}$ in 5 fractions (over 1 week) to 40 Gy in 15 fractions (over 3 weeks) was 1.16: well below the margin required to infer superiority to no radiotherapy.

TARGIT-IORT is inferior to WBRT in terms of local recurrence: in all four analyses, the 95\% CI for the excess local recurrence at 5 years with TARGIT-IORT does not include 0 (REF.'); and at a median follow-up duration of 